\title{
Three-dimensional upper limb movement characteristics in children and youth with dyskinetic cerebral palsy
}

I. Vanmechelen ${ }^{a}$, S. Bekteshi ${ }^{a}$, M. Konings ${ }^{a}$, H. Feys ${ }^{b}$, K. Desloovere $^{c}$, J-M. Aerts $^{d}$, E. Monbaliu $^{\text {a }}$

a KU Leuven Campus Bruges, Department of Rehabilitation Sciences, Bruges, Belgium b KU Leuven, Department of Rehabilitation Sciences, Leuven, Belgium c KU Leuven, Department of Rehabilitation Sciences, Pellenberg, Belgium d KU Leuven, Division Animal and Human Health Engineering, Leuven, Belgium

$\underline{\text { Introduction }}$

Dyskinetic cerebral palsy (DCP) is a condition characterized by abnormal postures or movements associated with impaired muscle tone regulation, movement control, and coordination (1). Threedimensional motion analysis has been frequently used to describe movement patterns in children with spastic CP, but less in children with DCP (2-4). Nevertheless, this could be an important contributor to understanding the complex movement patterns in children with DCP and subsequently, personalisation of treatment management (1).

\section{Research question}

1) Are the deviating movement characteristics in participants with DCP expressed in deviating joint angles and trajectory deviations? 2) Do participants with DCP have different speed characteristics during upper limb movements?

\section{Methodology}

Nine participants with DCP (mean age 17y 6m, range 11-25y) were included, with four bilateral participants leading to 13 DCP measurements. For comparison, 14 age-matched TD participants were included (mean age $17 \mathrm{y} 2 \mathrm{~m}$, range 12-23y). Participants were asked to perform 10 repetitions of three functional tasks: reaching forward (RF), reaching sideways (RS) and reach to grasp vertically (RGV). Seventeen reflective markers were placed over the body in 5 clusters: trunk, scapula, upper arm, forearm and hand. Static and dynamic calibrations were carried out to allow for joint angle calculations. Joint angle at point of task achievement, trajectory deviation, maximum velocity, timing of maximum velocity and movement smoothness were calculated. T-tests were used to assess the differences between TD children and children with DCP.

$\underline{\text { Results }}$

Participants with DCP showed significantly less elbow extension for all tasks, and significantly less elbow supination in the reach and grasp task. During reaching sideways, participants with DCP showed significantly higher external shoulder rotation, less arm abduction and higher external trunk rotation in comparison with their TD peers. Additionally, participants with DCP showed higher heterolateral trunk flexion during the reach and grasp task. For the spatio-temporal parameters, participants with DCP showed a significantly higher trajectory deviation and significantly lower smoothness and maximal speed during all tasks. For timing of maximum velocity, participants with DCP showed a significantly later peak velocity during the reach forwards task (table 1).

$\underline{\text { Discussion }}$

The decreased elbow extension during all tasks and the decreased elbow supination during the reach and grasp task agree with previous results in participants with spastic CP (2) and could be the result of the presence of spasticity in participants with DCP. The increased external trunk rotation during reach sideways could be a compensatory movement for the smaller shoulder abduction. Participants 
with DCP move less straight, where the presence of involuntary movements contributes to significantly higher trajectory deviations and significantly lower smoothness values for all tasks. Additionally, participants with DCP move slower in comparison with their TD peers, and they reach peak velocity at a later time point during the reach forward task.

\section{$\underline{\text { References }}$}

1. Monbaliu E, Himmelmann K, Lin J-P, Ortibus E, Bonouvrié L, Feys $\mathrm{H}$, et al. Clinical presentation and management of dyskinetic cerebral palsy. The Lancet Neurology. 2017;16(9):741-9. 2. Jaspers E, Desloovere K, Bruyninckx H, Klingels K, Molenaers G, Aertbelien E, et al. Threedimensional upper limb movement characteristics in children with hemiplegic cerebral palsy and typically developing children. Res Dev Disabil. 2011;32(6):2283-94.

3. Jaspers E, Feys H, Bruyninckx H, Cutti A, Harlaar J, Molenaers G, et al. The reliability of upper limb kinematics in children with hemiplegic cerebral palsy. Gait \& posture. 2011;33(4):568-75.

4. Butler EE, Rose J. The pediatric upper limb motion index and a temporal-spatial logistic regression: quantitative analysis of upper limb movement disorders during the Reach \& Grasp Cycle. Journal of biomechanics. 2012;45(6):945-51. 
Table 1: Mean and standard deviation of joint angles and spatio-temporal parameters for TD participants and participants with DCP

\section{REACH FORWARDS REACH AND GRASP VERTICAL REACH SIDEWAYS}

\begin{tabular}{|c|c|c|c|c|c|c|c|c|c|}
\hline & TD & DCP & $\begin{array}{c}p- \\
\text { value }\end{array}$ & TD & DCP & $\begin{array}{l}p- \\
\text { value }\end{array}$ & TD & DCP & $\begin{array}{l}\mathrm{p}- \\
\text { value }\end{array}$ \\
\hline \multicolumn{10}{|l|}{ JOINT ANGLES AT PTA } \\
\hline $\begin{array}{l}\text { TRUNK } \\
\text { FLEXION/EXTENSION }\end{array}$ & $\begin{array}{l}-3.11 \\
(5.59)\end{array}$ & $\begin{array}{l}-1.14 \\
(11.90)\end{array}$ & 0.583 & $\begin{array}{l}-2.94 \\
(5.97)\end{array}$ & $\begin{array}{l}-1.63 \\
(10.26)\end{array}$ & 0.687 & $\begin{array}{l}-1.09 \\
(7.44)\end{array}$ & $\begin{array}{l}0.37 \\
(9.55)\end{array}$ & 0.661 \\
\hline TRUNK AXIAL ROTATION & $\begin{array}{l}7.11 \\
(4.60)\end{array}$ & $\begin{array}{l}9.47 \\
(10.85)\end{array}$ & 0.109 & $\begin{array}{l}7.97 \\
(4.65)\end{array}$ & $\begin{array}{l}14.77 \\
(9.64)\end{array}$ & 0.264 & $\begin{array}{l}-8.17 \\
(4.68)\end{array}$ & $\begin{array}{l}-16.93 \\
(11.41)\end{array}$ & $0.014^{*}$ \\
\hline TRUNK LATERAL FLEXION & $\begin{array}{l}1.27 \\
(3.94)\end{array}$ & $\begin{array}{l}-1.64 \\
(5.13)\end{array}$ & 0.463 & $\begin{array}{l}0.18 \\
(3.74)\end{array}$ & $\begin{array}{l}-1.96 \\
(5.88)\end{array}$ & $0.026^{*}$ & $\begin{array}{l}1.02 \\
(4.56)\end{array}$ & $\begin{array}{l}1.75 \\
(6.00)\end{array}$ & 0.724 \\
\hline $\begin{array}{l}\text { SCAPULA } \\
\text { PRO/RETRACTION }\end{array}$ & $\begin{array}{l}45.15 \\
(9.40)\end{array}$ & $\begin{array}{l}41.70 \\
(11.88)\end{array}$ & 0.408 & $\begin{array}{l}45.56 \\
(8.13)\end{array}$ & $\begin{array}{l}43.37 \\
(10.19)\end{array}$ & 0.541 & $\begin{array}{l}7.73 \\
(12.25)\end{array}$ & $\begin{array}{l}10.81 \\
(14.31)\end{array}$ & 0.553 \\
\hline SCAPULA ROTATION & $\begin{array}{l}-23.78 \\
(11.23)\end{array}$ & $\begin{array}{l}-19.62 \\
(17.50)\end{array}$ & 0.467 & $\begin{array}{l}-17.14 \\
(10.94)\end{array}$ & $\begin{array}{l}-12.30 \\
(19.76)\end{array}$ & 0.259 & $\begin{array}{l}-23.01 \\
(13.77)\end{array}$ & $\begin{array}{l}-18.92 \\
(17.13)\end{array}$ & 0.499 \\
\hline SCAPULA TILTING & $\begin{array}{l}-2.76 \\
(8.68)\end{array}$ & $\begin{array}{l}4.74 \\
(13.93)\end{array}$ & 0.103 & $\begin{array}{l}-0.88 \\
(8.55)\end{array}$ & $\begin{array}{l}4.66 \\
(15.66)\end{array}$ & 0.273 & $\begin{array}{l}1.51 \\
(12.34)\end{array}$ & $\begin{array}{l}4.88 \\
(14.64)\end{array}$ & 0.522 \\
\hline ABDUCTION/ADDUCTION & $\begin{array}{l}92.55 \\
(9.04)\end{array}$ & $\begin{array}{l}84.33 \\
(9.74)\end{array}$ & 0.066 & $\begin{array}{l}90.71 \\
(9.63)\end{array}$ & $\begin{array}{l}83.38 \\
(10.47)\end{array}$ & 0.123 & $\begin{array}{l}8.44 \\
(10.31)\end{array}$ & $\begin{array}{l}21.44 \\
(16.54)\end{array}$ & $0.031^{*}$ \\
\hline SHOULDER ELEVATION & $\begin{array}{l}-84.63 \\
(11.06)\end{array}$ & $\begin{array}{l}-80.89 \\
(13.63)\end{array}$ & 0.439 & $\begin{array}{l}-78.19 \\
(10.71)\end{array}$ & $\begin{array}{l}-80.00 \\
(13.10)\end{array}$ & 0.697 & $\begin{array}{l}-74.86 \\
(11.29)\end{array}$ & $\begin{array}{l}-69.19 \\
(11.11)\end{array}$ & 0.201 \\
\hline SHOULDER ROTATION & $\begin{array}{l}-42.64 \\
(15.04)\end{array}$ & $\begin{array}{l}-51.44 \\
(13.47)\end{array}$ & 0.206 & $\begin{array}{l}-53.00 \\
(12.78)\end{array}$ & $\begin{array}{l}-55.96 \\
(46.85)\end{array}$ & 0.865 & $\begin{array}{l}-34.85 \\
(12.25)\end{array}$ & $\begin{array}{l}-50.49 \\
(18.37)\end{array}$ & $0.031^{*}$ \\
\hline $\begin{array}{l}\text { ELBOW FLEXION } \\
\text { EXTENSION }\end{array}$ & $\begin{array}{l}14.52 \\
(13.77)\end{array}$ & $\begin{array}{l}38.89 \\
(17.28)\end{array}$ & $0.000 *$ & $\begin{array}{l}22.039 \\
(13.46)\end{array}$ & $\begin{array}{l}39.40 \\
(16.58)\end{array}$ & $0.006^{*}$ & $\begin{array}{l}18.42 \\
(15.56)\end{array}$ & $\begin{array}{l}36.98 \\
(16.44)\end{array}$ & $0.006^{*}$ \\
\hline $\begin{array}{l}\text { ELBOW } \\
\text { PRO/SUPINATION }\end{array}$ & $\begin{array}{l}129.94 \\
(20.38)\end{array}$ & $\begin{array}{l}138.37 \\
(19.96)\end{array}$ & 0.288 & $\begin{array}{l}94.45 \\
(19.63)\end{array}$ & $\begin{array}{l}114.86 \\
(26.34)\end{array}$ & $0.030^{*}$ & $\begin{array}{l}123.74 \\
(21.98)\end{array}$ & $\begin{array}{l}134.53 \\
(22.96)\end{array}$ & 0.224 \\
\hline $\begin{array}{l}\text { WRIST } \\
\text { FLEXTION/EXTENSION }\end{array}$ & $\begin{array}{l}12.02 \\
(4.44)\end{array}$ & $\begin{array}{l}19.41 \\
(19.66)\end{array}$ & 0.182 & $\begin{array}{l}-14.57 \\
(14.69)\end{array}$ & $\begin{array}{l}-8.48 \\
(27.13)\end{array}$ & 0.470 & $\begin{array}{l}12.16 \\
(5.93)\end{array}$ & $\begin{array}{l}22.10 \\
(23.72)\end{array}$ & 0.141 \\
\hline WRIST DEVIATION & $\begin{array}{l}4.85 \\
(7.03)\end{array}$ & $\begin{array}{l}1.22 \\
(16.40)\end{array}$ & 0.456 & $\begin{array}{l}-1.19 \\
(9.73)\end{array}$ & $\begin{array}{l}-1.99 \\
(17.13)\end{array}$ & 0.881 & $\begin{array}{l}4.31 \\
(9.39)\end{array}$ & $\begin{array}{l}7.25 \\
(14.88)\end{array}$ & 0.541 \\
\hline \multicolumn{10}{|l|}{$\begin{array}{l}\text { SPATIO-TEMPORAL } \\
\text { PARAMETERS }\end{array}$} \\
\hline TRAJECOTRY DEVIATION & $\begin{array}{l}1.21 \\
(0.08)\end{array}$ & $\begin{array}{l}1.41 \\
(0.29)\end{array}$ & $0.000 *$ & $\begin{array}{l}1.07 \\
(0.03)\end{array}$ & $\begin{array}{l}1.29 \\
(0.24)\end{array}$ & $0.000 *$ & $\begin{array}{l}1.18 \\
(0.06)\end{array}$ & $\begin{array}{l}1.35 \\
(0.28)\end{array}$ & $0.000 *$ \\
\hline
\end{tabular}




\begin{tabular}{llllllllll}
\hline V MAX (m/s) & 1.29 & 1.00 & $0.000^{*}$ & 1.179 & 0.915 & $0.000^{*}$ & 1.60 & 1.24 & $0.000 *$ \\
& $(0.21)$ & $(0.23)$ & & $(0.22)$ & $(0.16)$ & & $(0.29)$ & $(0.36)$ \\
\hline TV MAX (\%) & 34 & 38 & $0.007^{*}$ & 36 & 38 & 0.363 & 38 & 39 & 0.205 \\
& $(3.45)$ & $(9.00)$ & & $(2.89)$ & $(12.17)$ & & $(3.69)$ & $(7.52)$ & -3.84 \\
\hline SMOOTHNESS & -3.11 & -3.61 & $0.000^{*}$ & -3.13 & -3.67 & $0.000^{*}$ & -3.25 & -3.84 & $0.000^{*}$ \\
& $(0.17)$ & $(0.46)$ & & $(0.19)$ & $(0.70)$ & & $(0.29)$ & $(0.54)$ \\
\hline
\end{tabular}

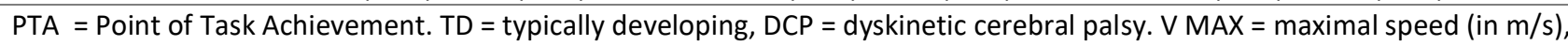

T V MAX = timing of maximum speed (in \% of reaching cycle). ${ }^{*} p<0.05$ 